\title{
Asymptotic Integration of Nonlinear Systems of Differential Equations whose Phase Portrait is Foliated on Invariant Tori
}

\author{
Yuri A. IL'IN \\ Faculty of Mathematics and Mechanics, St.-Petersburg State University, \\ Bibliotechnaja 2, St. Petergof, St.-Petersburg, 198804, Russia \\ E-mail: iljin@paloma.spbu.ru \\ Received February 8, 2000; Accepted March 12, 2000
}

\begin{abstract}
We consider the class of autonomous systems $\dot{x}=f(x)$, where $x \in \mathbf{R}^{2 n}, f \in C^{1}\left(\mathbf{R}^{2 n}\right)$ whose phase portrait is a Cartesian product of $n$ two-dimensional centres. We also consider perturbations of this system, namely $\dot{x}=f(x)+g(t, x)$, where $g \in C^{1}(\mathbf{R} \times$ $\mathbf{R}^{2 n}$ ) and $g$ is asymptotically small, that is $g \Rightarrow 0$ as $t \rightarrow+\infty$ uniformly with respect to $x$. The rate of decrease of $g$ is assumed to be $t^{-p}$ where $p>1$. We prove under this conditions the existence of bounded solutions of the perturbed system and discuss their convergence to solutions of the unperturbed system. This convergence depends on $p$. Moreover, we show that the original unperturbed system may be reduced to the form $\dot{r}=0, \dot{\theta}=A(r)$, and taking $r \in \mathbf{R}_{+}^{m}, \theta \in \mathbf{T}^{n}$, where $\mathbf{T}^{n}$ denotes the $n$-dimensional torus, we investigate the more general case of systems whose phase portrait is foliated on invariant tori. We notice that integrable Hamiltonian systems are of the same nature. We give also several examples, showing that the conditions of our theorems cannot be improved.
\end{abstract}

\section{Introduction}

A. In [3] the author investigated the asymptotic behaviour of solutions of an $\mathbf{R}^{2}$-system

$$
\dot{x}=f(x)+g(t, x),
$$

where the unperturbed system $\dot{x}=f(x)$ has a phase portrait like a linear centre and the perturbation $g(t, x) \Rightarrow 0$ as $t \rightarrow+\infty$. In this paper we give natural generalizations of this problem. 
B. First of all we consider an $\mathbf{R}^{2 n}$-system

$$
\left\{\begin{array}{ccc}
\dot{x}_{1} & = & f_{1}\left(x_{1}\right) \\
& \cdots \\
\dot{x}_{n} & =f_{n}\left(x_{n}\right),
\end{array}\right.
$$

where $x_{k} \in \mathbf{R}^{2}$ and $f_{k} \in \mathrm{C}^{1}\left(\mathbf{R}^{2}, \mathbf{R}^{1}\right)$ for all $k=1, \ldots, n$. We also assume that the phase portrait of each subsystem $\dot{x}_{k}=f_{k}\left(x_{k}\right)$ is similar to a linear centre, i.e. origin is a unique equilibrium point and the other trajectories are closed curves surrounding the origin. The phase portrait of the whole system (1.1) resembles the Cartesian product

$$
\left(\mathbf{R}_{+}^{1} \times \mathbf{S}^{1}\right) \times \cdots \times\left(\mathbf{R}_{+}^{1} \times \mathbf{S}^{1}\right)=\mathbf{R}_{+}^{n} \times \mathbf{T}^{n},
$$

where $\mathbf{R}_{+}^{1}=[0,+\infty)$ and $\mathbf{T}^{n}$ denotes the $n$-dimensional torus.

The first example of such a system is a linear system $\dot{x}=A x$ where all eigenvalues of matrix $A$ are pure imaginary and have only prime Jordan blocks. This system can be reduced by a linear transformation to a system of $n$ independent mathematical pendulums

$$
\left\{\begin{array}{ccc}
y_{1}^{\prime \prime}+a_{1} y_{1} & =0 \\
& \ldots \\
y_{n}^{\prime \prime}+a_{n} y_{n} & =0
\end{array}\right.
$$

where all $a_{k}>0$. The next more general example is a system of $n$ independent Duffing equations

$$
\left\{\begin{array}{cccc}
y_{1}^{\prime \prime}+a_{1} y_{1} & =h_{1}\left(y_{1}, y_{1}^{\prime}\right) \\
\cdots & \\
y_{n}^{\prime \prime}+a_{n} y_{n} & =h_{n}\left(y_{n}, y_{n}^{\prime}\right)
\end{array}\right.
$$

where every function $h_{k}$ does not destroy the centre defined by the linear part in each equation. We often meet the same systems in different applications of mathematics, mechanics, physics and engineering sciences. There are other important examples of similar systems, but we do not mention all of them here. The only exception is a Hamiltonian integrable system mentioned below.

We rewrite system (1.1) in brief form

$$
\dot{x}=f(x),
$$

where $x=\left(x_{1}, \ldots, x_{n}\right)^{\top}, f=\left(f_{1}, \ldots, f_{n}\right)^{\top}$. And we consider a perturbation of (1.1)

$$
\dot{x}=f(x)+g(t, x),
$$

where $g \in \mathrm{C}_{t, x}^{0,1}\left(\mathbf{R}_{t}^{1} \times \mathbf{R}_{x}^{2 n}, \mathbf{R}^{1}\right)$ and function $g$ satisfies the main assumption

(A1) $t^{p}\|g(t, x)\| \Rightarrow 0$ uniformly with respect to $x$ on compact subsets of $\mathbf{R}^{2 n}$ as $t \rightarrow \infty$.

An equivalent formulation of (A1) is:

(A2) there are positive continuous scalar functions $\alpha(s)$ and $\beta(t)$ such that $\alpha$ is increasing, $\beta$ is decreasing, $\lim _{t \rightarrow \infty} \beta(t)=0$ and

$$
t^{p}\|g(t, x)\| \leq \alpha(\|x\|) \beta(t) .
$$


The proof of this equivalence is standard enough and we omit it here. The following theorems are proved for system (1.3).

Theorem 4.2. If $p>1$ then system (1.3) has bounded solutions for $t \geq 0$.

Theorem 4.4. If $p>1$ then any bounded solution of (1.3) approaches (orbitally) a certain $n$-dimensional invariant torus of the unperturbed system (1.1).

Theorem 5.2. If $p>2$ then any bounded solution of (1.3) approaches a certain solution of unperturbed system (1.1).

It will be shown that for $p=1$ and $p=2$ these theorems will no longer be valid.

C. In Section 3 we construct for the system (1.1) action-angle variables ("quasi-polar" coordinates) in which (1.1) has the form $\dot{r}=0, \dot{\theta}=A(r)$ where $r=\left(r_{1}, \ldots, r_{n}\right)^{\top}$ are "quasi-polar" radii and $\theta=\left(\theta_{1}, \ldots, \theta_{n}\right)^{\top}$ are "quasi-polar" angles.

It gives us possibility to consider instead of (1.1) the more general system in the form

$$
\left\{\begin{array}{l}
\dot{r}=0 \\
\dot{\theta}=A(r)
\end{array}\right.
$$

where $r \in \mathbf{R}_{+}^{m}, \theta \in \mathbf{T}^{n}, A \in \mathrm{C}^{1}\left(\mathbf{R}_{+}^{m}, \mathbf{R}_{+}^{n} \backslash\{0\}\right)$ (i.e. each component $\left.A_{k}(r)>0\right)$. The phase portrait of (1.4) resembles the Cartesian product of a more complicated form than (1.2), namely

$$
\left(\mathbf{R}_{+}^{m_{1}} \times \mathbf{T}_{1}^{n}\right) \times \cdots \times\left(\mathbf{R}_{+}^{m_{d}} \times \mathbf{T}^{n_{d}}\right)=\mathbf{R}_{+}^{m} \times \mathbf{T}^{n}
$$

where $m_{1}+\cdots+m_{d}=m$ and $n_{1}+\cdots+n_{d}=n$. The perturbations of (1.4) need to be taken in the form

$$
\left\{\begin{array}{l}
\dot{r}=P(t, r, \theta) \\
\dot{\theta}=A(r)+Q(t, r, \theta)
\end{array}\right.
$$

where $P \in \mathrm{C}_{t, r, \theta}^{0,1,1}\left(\mathbf{R}^{1} \times \mathbf{R}_{+}^{m} \times \mathbf{T}^{n}, \mathbf{R}^{m}\right), Q \in \mathrm{C}_{t, r, \theta}^{0,1,1}\left(\mathbf{R}^{1} \times \mathbf{R}_{+}^{m} \times \mathbf{T}^{n}, \mathbf{R}^{n}\right.$ ) (Let us recall that $\theta \in \mathbf{T}^{n}$ means indeed that $\theta \in \mathbf{R}^{n}$ and functions $P$ and $Q$ are periodic in each component of the vector $\theta$.)

We shall suppose that $P$ and $Q$ satisfy the following main assumption

(A3) $t^{p}\|P(t, r, \theta)\|, t^{p}\|Q(t, r, \theta)\| \Rightarrow 0$ uniformly with respect to $r$ on compact subsets of $\mathbf{R}_{+}^{m}$ and for all $\theta \in \mathbf{T}^{n}$ as $t \rightarrow \infty$.

An equivalent formulation of (A3) is:

(A4) there are positive continuous scalar functions $\alpha(s)$ and $\beta(t)$ such that $\alpha$ is increasing, $\beta$ is decreasing, $\lim _{t \rightarrow \infty} \beta(t)=0$ and

$$
t^{p}\|P(t, r, \theta)\|, t^{p}\|Q(t, r, \theta)\| \leq \alpha(\|r\|) \beta(t) .
$$

We prove the following theorems for (1.5).

Theorem 4.1. If $p>1$ then system (1.5) has solutions bounded with respect to $r$-coordinates for $t \geq 0$.

Theorem 4.3. If $p>1$ then any bounded solution of (1.5) approaches (orbitally) a certain invariant torus of the unperturbed system (1.4). 
Theorem 5.1. If $p>2$ then any bounded solution of (1.5) converges to a certain solution of the unperturbed system (1.4).

We note at the end of this section that problems of asymptotic integration are classical in the theory of differential equations. If we eliminate the $\theta$-equation and the variable $\theta$ from (1.5) we obtain a well-investigated in the literature problem of the perturbations of zero system (see [5]- [7], monographs [1] and [8] give the general theory). From this point of view Theorems 4.1-4.4 are quite natural and expected statements. On the contrary, Theorems 5.1-5.2 represent more delicate results. We consider the construction of the "quasi-polar" variables and proof of Theorems 5.1-5.2 as one of the most important parts of our paper.

\section{The existence of a positively-definite integral}

The aim of this section is to prove the following statement.

Theorem 2.1. Let $\dot{x}_{k}=f_{k}\left(x_{k}\right)$ be a two-dimensional subsystem of (1.1). Then it has a differentiable positively-definite integral $U_{k}\left(x_{k}\right)$ on $\mathbf{R}^{2}$.

This is a known result. But because we shall need further some detailed properties of $U_{k}$ we give an explicit proof of this theorem.

Proof. For convenience we omit the index $k$ and will write simply $x$ and $f$ instead of $x_{k}$ and $f_{k}$. We belief that no confusion with the system (1.1) can arise. Let $x=\left(z_{1}, z_{2}\right)^{\top}$ and $f=\left(h_{1}, h_{2}\right)^{\top}$. The system $\dot{x}=f(x)$ can be rewritten in the form

$$
\dot{z}_{1}=h_{1}\left(z_{1}, z_{2}\right), \quad \dot{z}_{2}=h_{2}\left(z_{1}, z_{2}\right) .
$$

Consider the system "orthogonal" to $(2.1)$

$$
\dot{z}_{1}=-h_{2}\left(z_{1}, z_{2}\right), \quad \dot{z}_{2}=h_{1}\left(z_{1}, z_{2}\right) \text {. }
$$

It is evident that the right-hand vector fields of (2.1) and (2.2) are orthogonal at each point. This implies that (2.2) has also a unique equilibrium point at the origin and other trajectories of (2.2) cross orthogonally all cycles of (2.1) at a unique point. For if any trajectory of (2.2) intersects some cycle of (2.1) at more than one point then there is a point on this cycle where right-hand vectors of (2.1) and (2.2) will tangent each other.

Let $\xi(t)$ be a certain nonzero solution of $(2.2)$ and $I=(a, b)$ denote its maximal interval of existence. Let $\Gamma$ denote the trajectory of $\xi(t)$. Without loss of generality we can assume that $\Gamma$ crosses the cycles of (2.1) from inside to outside with increase of $t$ (otherwise one needs to consider the system $\dot{z}_{1}=h_{2}, \dot{z}_{2}=-h_{1}$ instead of $(2.2)$ ). It follows from PoincaréBendixon theory that $\xi(t) \rightarrow 0$ as $t \rightarrow a_{+}$and $\|\xi(t)\| \rightarrow \infty$ as $t \rightarrow b_{-}$. Indeed, system (2.2) cannot have closed curves (cycles) because then they must intersect the cycles of (2.1) at more than one point. Hence the $\alpha$-limit set of $\xi(t)$ is not empty and may consist of only origin. By the same reasons the $\omega$-limit set of $\xi(t)$ must be empty, i.e. $\|\xi(t)\| \rightarrow \infty$ as $t \rightarrow b_{-}$. It is clear that when time $t$ increases from $a$ to $b$ the solution $\xi(t)$ crosses each cycle of (2.1). We have also that $a=-\infty$ because $\xi(t)$ remains bounded as $t \rightarrow a_{+}$. 
Let $\varphi\left(t, x^{0}\right)$ denote the solution of $(2.1)$ with initial condition $\varphi\left(0, x^{0}\right)=x^{0}$. We define the mapping $\chi: \mathbf{R}^{2} \backslash\{0\} \rightarrow \Gamma$ by the formula

$$
\chi(x)=\{\varphi(t, x): t \in \mathbf{R}\} \cap \Gamma=\varphi(\mathbf{R}, x) \cap \Gamma .
$$

It is clear that $\chi \in \mathrm{C}^{1}$ because the cycle $\varphi(\mathbf{R}, x)$ intersects $\Gamma$ transversally. Let $\xi^{-1}: \Gamma \rightarrow I$ be the mapping which to every $x \in \Gamma$ associates the time $t$ such that $x=\xi(t)$. Since $\xi(t)$ is differentiable and $\xi^{\prime}(t) \neq 0$ (it is not equilibrium point) we have $\xi^{-1} \in \mathrm{C}^{1}(\Gamma, I)$. Finally we define on $\mathbf{R}^{2} \backslash\{0\}$ the function $U_{1}(x)$ by the equality

$$
U_{1}(x)=\left(\xi^{-1} \circ \chi\right)(x) .
$$

We point out some properties of $U_{1}$.

1) $U_{1} \in \mathrm{C}^{1}\left(\mathbf{R}^{2} \backslash\{0\}, I\right)$,

2) $U_{1}(\xi(t))=t$

3) $U_{1}(\varphi(t, x)) \equiv U_{1}(x)$, thus $U_{1}$ is an integral for system $(2.1)$ on $\mathbf{R}^{2} \backslash\{0\}$,

4) equation $U_{1}(x)=r$ defines a unique cycle of (2.1)

(indeed, curve $\xi(t)$ intersects all cycles of (2.1) with increasing $t$ at only one point; hence $U_{1}$ monotonically increases along $\Gamma$; therefore each level curve of $U_{1}(x)$ consists of a unique cycle).

5) $\lim _{x \rightarrow 0} U_{1}(x)=-\infty$. (Indeed, $\left.x \rightarrow 0 \Rightarrow \chi(x) \rightarrow 0 \Rightarrow\left(\xi^{-1} \circ \chi\right)(x) \rightarrow a=-\infty\right)$.

By setting $U_{2}(x)=\exp \left(U_{1}(x)\right)$ we construct a continuous positively-definite integral for $(2.1)$ on whole $\mathbf{R}^{2}$ (we assume naturally $U_{2}(0)=0$ ). It is clear that $U_{2}\left(\mathbf{R}^{2}\right)=\left[0, e^{b}\right)$. Since differentiability of $U_{2}$ can be broken only at $x=0$ let us consider the behaviour of the gradient $D U_{2}(x)$ as $x \rightarrow 0$. There are two possibilities.

1) $\limsup _{x \rightarrow 0}\left\|D U_{2}(x)\right\|<\infty$.

2) $\limsup _{x \rightarrow 0}\left\|D U_{2}(x)\right\|=\infty$.

In the first case we define $U(x)=U_{2}^{2}(x)$. We thus have $\lim _{x \rightarrow 0} D U(x)$ $=2 \lim _{x \rightarrow 0} U_{2}(x) D U_{2}(x)=0$ and $U(x)$ is therefore the required integral for (2.1).

In the second case we will construct the integral $U(x)$ in the form $\left(\mu \circ U_{2}\right)(x)$ where a scalar function $\mu$ is chosen to smooth out the discontinuity of $D U_{2}$ at origin. To do this we first define the function $\nu(s)=\sup _{x \in\left(U_{2}\right)^{-1}(s)}\left\|D U_{2}(x)\right\|$ for $s \in\left(0, e^{b}\right)$. We have $\nu \in \mathrm{C}\left(0, e^{b}\right)$, since the sets $\left(U_{2}\right)^{-1}(s)$ are compact and depend continuously on $s$. The following properties are evident:

1) $\nu(s) \geq 0$.

2) $\lim _{s \rightarrow+0} \nu(s)=\infty$.

3) $\nu\left(U_{2}(x)\right) \geq\left\|D U_{2}(x)\right\|$. 
Finally we define $\mu(s)=\int_{0}^{s}(1+\nu(u))^{-2} d u$. It is easy to see that $\mu \in \mathrm{C}^{1}\left[0, e^{b}\right)$ and $\mu(0)=0$. We set $U(x)=\mu\left(U_{2}(x)\right)$ and verify that $U(x)$ is the required integral for (2.1). All we need is to check that $D U(x)$ exists at $x=0$. But it is fulfilled, because

$$
\begin{aligned}
\lim _{x \rightarrow 0} & \|D U(x)\|=\lim _{x \rightarrow 0}\left\|\mu^{\prime}\left(U_{2}(x)\right) D U_{2}(x)\right\| \\
& =\lim _{x \rightarrow 0}\left\|\frac{D U_{2}(x)}{\left(1+\nu\left(U_{2}(x)\right)\right)^{2}}\right\| \stackrel{\text { (property } 3)}{\leq} \lim _{x \rightarrow 0}\left(\frac{1}{1+\left\|D U_{2}(x)\right\|}\right)=0 .
\end{aligned}
$$

We point out some properties of $U$.

1) $U \in \mathrm{C}^{1}\left(\mathbf{R}^{2},\left[0, b^{*}\right)\right)$, where $b^{*}=e^{2 b}$ or $\mu\left(e^{b}\right)$.

2) $U(x)=0 \Leftrightarrow x=0$, i.e. $U$ is positively definite.

3) $U$ monotonically increases along $\Gamma: \quad \partial U / \partial \Gamma>0$.

4) each level curve $U(x)=r$ defines a unique cycle of (2.1).

The theorem is proved.

Note 2.1. For simplicity we will further assume that $b^{*}=+\infty$ because it is easy to construct a $\mathrm{C}^{1}$-function $\gamma:\left[0, b^{*}\right) \rightarrow[0,+\infty)$ and consider the new integral $\gamma(U(x))$ instead of $U(x)$.

\section{The action-angle or quasi-polar coordinates}

In this section we construct new coordinates for more convenient notation of systems (1.1) and (1.3). This procedure is a standard one for Hamiltonian systems, see for example the textbook 河. But we would notice that our coordinates are not identical to the hamiltonian action-angle coordinates. We introduce them in a more complicated and sly way, so that the change of variables does not break the asymptotic properties of the perturbations and solutions.

To each $\mathbf{R}^{2}$-component $x_{k}$ of vector $x$ we put in correspondence two scalar coordinates $r_{k}$ and $\theta_{k}$. We define the first "quasi-polar" coordinate (the "radius") $r_{k}$ by $r_{k}=U_{k}\left(x_{k}\right)$. In accordance to Section 2, the equation $U_{k}\left(x_{k}\right)=r_{k}$ for each $r_{k}>0$ defines a unique cycle of $\dot{x}_{k}=f_{k}\left(x_{k}\right)$. Moreover the function $r_{k}=U_{k}\left(x_{k}\right)$ is invertible on $\Gamma$, since $U_{k}$ monotonically increases along $\Gamma$. Let $v_{k}\left(r_{k}\right)$ denote this inversion. We have

1) $v_{k} \in \mathrm{C}^{1}\left(\mathbf{R}_{+}, \Gamma\right)$,

2) $v_{k}\left(r_{k}\right)=\Gamma \cap\left\{x_{k}: U_{k}\left(x_{k}\right)=r_{k}\right\}$,

3) $U_{k}\left(v_{k}\left(r_{k}\right)\right)=r_{k}$.

We denote the period of any cycle $U_{k}\left(x_{k}\right)=r_{k}$ by $T_{k}\left(r_{k}\right)$. It is equal to the time for the next intersection with $\Gamma$ of the solution passing at $t=0$ through the point $x_{k}=v_{k}\left(r_{k}\right)$. Since $\Gamma$ is a tranversal, we have $T_{k} \in \mathrm{C}^{1}$. We now prove the following

Proposition 3.1. The value $1 / T_{k}\left(r_{k}\right)$ is well-defined on $\mathbf{R}_{+}$. 
Proof. Obviously, $T_{k}\left(r_{k}\right) \neq 0$ for $r_{k} \neq 0$. It remains to prove that $T_{k}(0) \neq 0$. Let us use the notations from the proof of Theorem 2.1. Changing in (2.1) coordinates $z_{1}$ and $z_{2}$ into the standard polar coordinates $z_{1}=\rho \cos \alpha, z_{2}=\rho \sin \alpha$, we get for $\alpha$

$$
\dot{\alpha}=\rho^{-1}\left(\cos \alpha h_{2}(\rho \cos \alpha, \rho \sin \alpha)-\sin \alpha h_{1}(\rho \cos \alpha, \rho \sin \alpha)\right)=\rho^{-1} F(\rho, \alpha) .
$$

Obviously, $F \in \mathrm{C}^{1}\left(\mathbf{R}_{+} \times \mathbf{R}\right)$ and $F(0, \alpha) \equiv 0$. Then Hadamard's lemma implies $F(\rho, \alpha) \equiv$ $\rho G(\rho, \alpha)$ where $G \in \mathrm{C}\left(\mathbf{R}_{+} \times \mathbf{R}\right)$. Hence, $\dot{\alpha}=G(\rho, \alpha)$. Let $(\rho(t), \alpha(t))$ denote the solution passing through the initial point $\left(\rho_{0}, 0\right)$ at $t=0$. Let $T\left(\rho_{0}\right)$ be the period of it. It is clear that $\alpha\left(T\left(\rho_{0}\right)\right)=2 \pi$. On other hand, $\alpha\left(T\left(\rho_{0}\right)\right)=\int_{0}^{T\left(\rho_{0}\right)} G(\rho(t), \alpha(t)) d t$. For any $\rho_{0} \in[0,1]$ the solution $(\rho(t), \alpha(t))$ belongs to a certain compact set, therefore $G(\rho(t), \alpha(t))$ is uniformly bounded with respect to $\rho_{0}$. If $T\left(\rho_{0}\right) \rightarrow 0$ as $\rho_{0} \rightarrow 0$, then

$$
2 \pi=\lim _{T\left(\rho_{0}\right) \rightarrow 0} \alpha\left(T\left(\rho_{0}\right)\right)=\lim _{T\left(\rho_{0}\right) \rightarrow 0} \int_{0}^{T\left(\rho_{0}\right)} G(\rho(t), \alpha(t)) d t=0 .
$$

This contradiction proves the proposition.

Change time in the system $\dot{x}_{k}=f_{k}\left(x_{k}\right)$ by formula

$$
t=T_{k}\left(r_{k}\right) \tau .
$$

Since $\dot{r}_{k}=0$ along the solutions we have $d t=T_{k}\left(r_{k}\right) d \tau$ and

$$
\frac{d x_{k}}{d t}=T_{k}\left(r_{k}\right) f_{k}\left(x_{k}\right)
$$

All solutions of (3.1) have the common period 1, thus the centre defined by (3.1) is an isochronous one. Let $\psi_{k}\left(\tau, x_{k}\right)$ be the solution of (3.1) satisfying the initial condition $\psi_{k}\left(0, x_{k}\right)=x_{k}$. We define the second "quasi-polar" coordinate (the "angle") $\theta_{k}$ in such a way that $\psi_{k}\left(-\theta_{k}, x_{k}\right) \in \Gamma$. I.e. $-\theta_{k}$ is the time of the first intersection of solution $\psi_{k}\left(\tau, x_{k}\right)$ with $\Gamma$. It is clear that $\theta_{k}$ depends smoothly on $x_{k}$. Thus the general formula for the transformation to the new coordinates is

$$
x_{k}=\psi_{k}\left(\theta_{k}, v_{k}\left(r_{k}\right)\right) .
$$

Let us investigate some properties of the "quasi-polar" variables. For any function $h(x, y)$ the notations $D_{1} h$ and $D_{2} h$ denote the partial derivatives of $h$ with respect to the first and second argument correspondingly. Let $J_{k}\left(r_{k}, \theta_{k}\right)$ be the Jacobian matrix of the change of variables (3.2). We have

$$
J_{k}\left(r_{k}, \theta_{k}\right)=\frac{D \psi_{k}\left(\theta_{k}, v_{k}\left(r_{k}\right)\right)}{D\left(r_{k}, \theta_{k}\right)}=\left[D_{2} \psi_{k}\left(\theta_{k}, v_{k}\left(r_{k}\right)\right) v_{k}^{\prime}\left(r_{k}\right), \quad D_{1} \psi_{k}\left(\theta_{k}, v_{k}\left(r_{k}\right)\right)\right] .
$$

Since $\psi_{k}\left(\theta_{k}+1, v_{k}\left(r_{k}\right)\right) \equiv \psi_{k}\left(\theta_{k}, v_{k}\left(r_{k}\right)\right)$, the matrix $D_{2} \psi_{k}\left(\theta_{k}, v_{k}\left(r_{k}\right)\right)$ is 1-periodic with respect to $\theta_{k}$. On the other hand $D_{2} \psi_{k}$ is the derivative of the solution $\psi_{k}$ with respect to initial values, i.e. it is a fundamental matrix of a so-called linear system in variation. Hence $D_{2} \psi_{k}$ is nonsingular and $D_{2} \psi_{k}\left(0, v_{k}\left(r_{k}\right)\right)=E$. It follows from periodicity that the values

$$
\left\|D_{2} \psi_{k}\left(\theta_{k}, v_{k}\left(r_{k}\right)\right)\right\|, \quad\left\|\left(D_{2} \psi_{k}\left(\theta_{k}, v_{k}\left(r_{k}\right)\right)\right)^{-1}\right\|, \quad \operatorname{det} D_{2} \psi_{k}\left(\theta_{k}, v_{k}\left(r_{k}\right)\right)
$$


and $\operatorname{det}\left(D_{2} \psi_{k}\left(\theta_{k}, v_{k}\left(r_{k}\right)\right)\right)^{-1}$ are bounded away from zero and infinity for each fixed $r_{k}$ and $\forall \theta_{k} \in \mathbf{R}$.

Since $\psi_{k}\left(\theta_{k}, v_{k}\left(r_{k}\right)\right)$ is a solution of (3.1), we have

$$
D_{1} \psi_{k}\left(\theta_{k}, v_{k}\left(r_{k}\right)\right)=T_{k}\left(r_{k}\right) f_{k}\left(\psi_{k}\left(\theta_{k}, v_{k}\left(r_{k}\right)\right)\right) \text {. }
$$

This yields $J_{k}\left(r_{k}, 0\right)=\left[E \cdot v_{k}^{\prime}\left(r_{k}\right), T_{k}\left(r_{k}\right) f_{k}\left(v_{k}\left(r_{k}\right)\right)\right]$. But derivative $v_{k}^{\prime}$ is a tangent vector to $\Gamma$ at the point $v_{k}\left(r_{k}\right)$, because $v_{k}\left(r_{k}\right)$ is some parametrization of curve $\Gamma$. Hence $v_{k}^{\prime}\left(r_{k}\right)$ is to be orthogonal to the vector $f_{k}\left(v_{k}\left(r_{k}\right)\right)$. Thus matrix $J_{k}\left(r_{k}, 0\right)$ is non singular $\forall r_{k}>0$.

It is easy to check the following statement. Let $\dot{y}=F(y)$ be an arbitrary $C^{1}$-smooth system and $\varphi(t, y)$ be the solution of it with initial condition $\varphi(0, y)=y$, then $F(\varphi(t, y))=$ $D_{2} \varphi(t, y) F(y)$.

This implies $T_{k}\left(r_{k}\right) f_{k}\left(\psi_{k}\left(\theta_{k}, v_{k}\left(r_{k}\right)\right)\right)=D_{2} \psi_{k}\left(\theta_{k}, v_{k}\left(r_{k}\right)\right) T_{k}\left(r_{k}\right) f_{k}\left(v_{k}\left(r_{k}\right)\right)$ and therefore

$$
\begin{aligned}
& J_{k}\left(r_{k}, \theta_{k}\right)=\left[\left(D_{2} \psi_{k}\right) v_{k}^{\prime}, \quad\left(D_{2} \psi_{k}\right) T_{k} f_{k}\right]=\left(D_{2} \psi_{k}\right)\left[\begin{array}{ll}
v_{k}^{\prime}, & T_{k} f_{k}
\end{array}\right] \\
& =D_{2} \psi_{k}\left(\theta_{k}, v_{k}\left(r_{k}\right)\right) J_{k}\left(r_{k}, 0\right) \text {. }
\end{aligned}
$$

Each matrix in the last expression is nonsingular and possesses "good" properties. So this equality guarantees that the Jacobian matrix $J_{k}$ is nonsingular for fixed $r_{k}>0$ and arbitrary $\theta_{k} \in \mathbf{R}$. Moreover, $\operatorname{det} J_{k}$, $\operatorname{det} J_{k}^{-1},\left\|J_{k}\right\|$ and $\left\|J_{k}^{-1}\right\|$ are bounded away from zero and infinity. Similar properties of boundedness will also be fulfilled if we take $r_{k} \in K$ where $K$ is some compact set of $\mathbf{R}_{+}$and $\theta_{k}$ as before will be an arbitrary one.

In the new variables system (3.1) will have the following form

$$
\frac{d r_{k}}{d \tau}=0, \quad \frac{d \theta_{k}}{d \tau}=1
$$

The original system $\dot{x}_{k}=f_{k}\left(x_{k}\right)$ is transformed into

$$
\dot{r}_{k}=0, \quad \dot{\theta}_{k}=1 / T_{k}\left(r_{k}\right)
$$

(replacing $\left.d t=T_{k}\left(r_{k}\right) d \tau\right)$.

Finally the full system (1.1) is transformed into

$$
\dot{r}=0, \quad \dot{\theta}=A(r),
$$

where $r=\left(r_{1}, \ldots, r_{n}\right)^{\top}, \theta=\left(\theta_{1}, \ldots, \theta_{n}\right)^{\top}, A(r)=\left(A_{1}\left(r_{1}\right), \ldots, A_{n}\left(r_{n}\right)\right)^{\top}, A_{k}\left(r_{k}\right)=$ $1 / T_{k}\left(r_{k}\right)$.

Changing the variables in the perturbed system (1.3), we obtain

$$
\left(\begin{array}{c}
\dot{r} \\
\dot{\theta}
\end{array}\right)=\left(\begin{array}{c}
0 \\
A(r)
\end{array}\right)+[J(r, \theta)]^{-1} g(t, \psi(\theta, v(r)))=\left(\begin{array}{c}
0 \\
A(r)
\end{array}\right)+\left(\begin{array}{c}
P(t, r, \theta) \\
Q(t, r, \theta)
\end{array}\right)
$$

(we use natural notations $J=\operatorname{diag}\left(J_{1}, \ldots, J_{n}\right), \psi=\left(\psi_{1}, \ldots, \psi_{n}\right)^{\top}$, $\left.v(r)=\left(v_{1}\left(r_{1}\right), \ldots, v_{n}\left(r_{n}\right)\right)^{\top}\right)$. The functions $P$ and $Q$ possess the following properties:

1) they are in $C_{t, r, \theta}^{0,1,1}$,

2) they are 1-periodic with respect to each components of $\theta$, 
3) if $g$ satisfies the assumptions (A1) (or (A2)) then $P$ and $Q$ satisfy (A3) (or (A4)).

Indeed, let $K \subset \mathbf{R}_{+}^{n}$ be a compact set and $r \in K$. There exist $M>0$ such that $\left\|(J(r, \theta))^{-1}\right\| \leq M$ for all $r \in K$ and $\theta \in \mathbf{R}^{n}$. Hence

$$
t^{p}\left\|\left(\begin{array}{c}
P(t, r, \theta) \\
Q(t, r, \theta)
\end{array}\right)\right\| \leq M t^{p}\|g(t, \psi(\theta, v(r)))\| \Rightarrow 0 \quad \text { as } \quad t \rightarrow+\infty .
$$

(we note that if $r \in K$ then cycle $\psi(\theta, v(r))$ does not leave some compact set in $\mathbf{R}^{2 n}$ for all $\left.\theta \in \mathbf{R}^{n}\right)$.

Thus, we have shown that systems (1.1) and (1.3) may be reduced to systems (1.4) and (1.5) while preserving assumption (A3) (or (A4)).

Note 3.1. We want to underline that it is not so difficult to reduce formally system (1.3) to (1.5) (see theory of Hamiltonian system in 四). The problem is to keep the asymptotic properties (A1-A2) as well as the boundedness and covergence of solutions under the inverse transformation. For this we use the trick with isochronicity. Without it the Jacobian matrix may be unbounded and this manner of reasoning fails.

\section{The existence of bounded solutions}

Let us consider the system (1.5).

Theorem 4.1. Let $P$ satisfy assumption (A3) or (A4) with $p>1$. Then (1.5) has solutions bounded with respect to $r$-coordinates.

Proof. We shall find a comparison equation for $\|r\|$. We have

$$
\frac{d}{d t}\|r\|^{2}=\frac{d}{d t}(r, r)=2(r, \dot{r})=2(r, P(t, r, \theta)),
$$

here $(\cdot, \cdot)$ denotes inner product. This gives for $\|r\| \neq 0$

$$
\frac{d}{d t}\|r\| \leq\|P(t, r, \theta)\| \stackrel{(A 4)}{\leq} \alpha(\|r\|) \beta(t) / t^{p} \leq \alpha(\|r\|) / t^{p},
$$

(we can assume that $\beta(t) \leq 1$ for sufficiently large $t$, because $\beta(t) \rightarrow 0$ ). Let us consider the comparison equation

$$
\frac{d}{d t} \rho=\alpha(\rho) / t^{p}
$$

It is well-known (see [1]) that every function $r(t)$ satisfying inequality (4.1) does not exceed the solution $\rho(t)$ of (4.2) with initial condition $\rho\left(t_{0}\right)=\left\|r\left(t_{0}\right)\right\|$. Thus the existence of bounded solutions of (4.2) will imply the same for (1.5).

Let $G(\rho)=\int_{1}^{\rho} 1 / \alpha(s) d s$. Since $G^{\prime}(\rho)>0$, function $G(\rho)$ is monotone increasing and invertible. The solution of (4.2) with initial condition $\rho\left(t_{0}\right)=\rho_{0}$ has the form

$$
G(\rho(t))-G\left(\rho_{0}\right)=(p-1)\left(t_{0}^{1-p}-t^{1-p}\right) .
$$

There are two possibilities. 
A. $\lim _{\rho \rightarrow \infty} G(\rho)=+\infty$. Then all solutions of (4.2) must be bounded. For if some solution $\rho(t) \rightarrow+\infty$ as $t \rightarrow+\infty$, then the left-hand side of (4.3) tends to $\infty$ whereas the right-hand side tends to $(p-1) t_{0}^{1-p}<\infty$. We got a contradiction.

B. $\lim _{\rho \rightarrow \infty} G(\rho)=G^{*}<\infty$. Fix $\rho_{0}>0$ and take $t_{0}$ so large that $G^{*}-G\left(\rho_{0}\right)>$ $(p-1) t_{0}^{1-p}$. Then the solution of (4.2) satisfying the initial condition $\rho\left(t_{0}\right)=\rho_{0}$ is bounded. Otherwise, tending $t \rightarrow+\infty$ in (4.3) and assuming that $\rho(t) \rightarrow+\infty$ we get the impossible equality $G^{*}-G\left(\rho_{0}\right)=(p-1) t_{0}^{1-p}$.

Thus the equation (4.2) has bounded solutions in both cases. So the theorem is proved.

Note 4.1. The case $\mathbf{A}$ occurs when $P$ satisfies the well-known inequality (see [1])

$$
\|P(t, r, \theta)\| \leq h(\|r\|) \gamma(t),
$$

where $\int^{\infty} \gamma(t) d t<\infty$ and $\int^{\infty} 1 / h(s) d s=\infty$. In this case all solutions of (1.5) are bounded with respect to $r$. Conversely, there are unbounded solutions in the case $\mathbf{B}$ as the next example shows.

Example 4.1. Consider the system $\dot{r}=0, \dot{\theta}=1$ and its perturbation $\dot{r}=r^{2} / t^{2}, \dot{\theta}=1$. The perturbed system has unbounded extendable to infinity solution $r=t$. All solutions lying inside the sector $0<r<t$ are bounded and those lying outside it are unbounded (moreover, they are unextendable to infinity).

Note 4.2. The condition $p>1$ in Theorem 4.1 cannot be generalized to include the case $p=1$. See Example 4.2 below.

Now we give reformulations of Theorem 4.1 and Note 2.1 for system (1.3).

Theorem 4.2. Suppose that perturbation $g$ in (1.3) satisfies (A1) or (A2) with $p>1$. Then (1.3) has bounded solutions.

Proof. As it follows from Section 3 the system (1.3) written in "quasi-polar" coordinates satisfies all conditions of Theorem 4.1.

Note 4.3. All solutions of (1.3) are bounded if $\|g(t, x)\| \leq h(\|x\|) \gamma(t)$, where $\int^{\infty} \gamma(t) d t<$ $\infty$ and $\int^{\infty} 1 / h(s) d s=\infty$.

Example 4.2. The present example shows that condition $p>1$ cannot be generalized to include the case $p=1$. We take the standard two-dimensional centre

$$
\dot{x}_{1}=-x_{2}, \quad \dot{x}_{2}=x_{1},
$$

and perturb it in the following way

$$
\dot{x}_{1}=-x_{2}+\frac{\cos t}{t \ln t}, \quad \dot{x}_{2}=x_{1}+\frac{\sin t}{t \ln t}, \quad(t>1) .
$$

It is evident that $\cos t /(t \ln t)$ and $\sin t /(t \ln t)$ satisfy $(\mathrm{A} 2)$ with $p=1, \alpha(s)=1, \beta(t)=$ $1 / \ln t$. But all solutions of the perturbed system have the form

$$
x_{1}=\left(C_{1}+\ln (\ln t)\right) \cos t-C_{2} \sin t, \quad x_{2}=\left(C_{1}+\ln (\ln t)\right) \cos t+C_{2} \sin t,
$$

and are unbounded. 
Yu.A. Il'in

Now we return to system (1.5) and show that under the assumptions of Theorem 4.1 every bounded solution approaches a certain invariant torus of (1.4).

Theorem 4.3. Let $(r(t), \theta(t))$ be a bounded solution of (1.5) with respect to $r$-coordinates and

$$
t^{p}\|P(t, r(t), \theta(t))\| \rightarrow 0 \quad \text { as } \quad t \rightarrow 0
$$

with $p>1$. There exists $r^{*}$ such that

$$
\lim _{t \rightarrow+\infty} r(t)=r^{*}
$$

Comment. It means that solution $(r(t), \theta(t))$ approaches the invariant torus $r=r^{*}$ of (1.4).

Note 4.4. Evidently, if (A3) or (A4) holds then every bounded solution of (1.5) satisfies ( $\tilde{A} 3)$.

Proof. We have

$$
r(t)=r\left(t_{0}\right)+\int_{t_{0}}^{t} \dot{r}(s) d s=r\left(t_{0}\right)+\int_{t_{0}}^{t} P(s, r(s), \theta(s)) d s .
$$

Assumption $(\tilde{A} 3)$ implies that $\int_{t_{0}}^{\infty}\|P(s, r(s), \theta(s))\| d s$ converges. Hence the limit

$$
\lim _{t \rightarrow+\infty} r(t)=r\left(t_{0}\right)+\int_{t_{0}}^{\infty} P(s, r(s), \theta(s)) d s=r^{*}
$$

exists. The theorem is proved.

Note 4.5. The condition $p>1$ cannot be generalized to include the case $p=1$, see Example 4.4.

Note 4.6. The next example demonstrates that assumptions of Theorem 4.3 don't imply even orbital convergence of bounded solutions of (1.5) to certain solution of (1.4). However this convergence occurs if $\theta \in \mathbf{R}^{1}$ (see [3]), see also Note 5.2.

Example 4.3. Consider the following system

$$
\dot{r}_{1}=0, \quad \dot{r}_{2}=0, \quad \dot{\theta}_{1}=r_{1}, \quad \dot{\theta}_{2}=r_{2},
$$

and its perturbation

$$
\dot{\bar{r}}_{1}=1 / t^{2}, \quad \dot{\bar{r}}_{2}=2 / t^{2}, \quad \dot{\bar{\theta}}_{1}=\bar{r}_{1}, \quad \dot{\bar{\theta}}_{2}=\bar{r}_{2},
$$

Solutions of (4.5) are

$$
r_{1}=r_{1}^{0}, \quad r_{2}=r_{2}^{0}, \quad \theta_{1}=r_{1}^{0} t+\theta_{1}^{0}, \quad \theta_{2}=r_{2}^{0} t+\theta_{2}^{0},
$$


where $r_{1}^{0}, r_{2}^{0}, \theta_{1}^{0}, \theta_{2}^{0}$ are arbitrary constants (initial values). Solutions of (4.6) are

$$
\bar{r}_{1}=\bar{r}_{1}^{0}-1 / t, \quad \bar{r}_{2}=\bar{r}_{2}^{0}-2 / t, \quad \bar{\theta}_{1}=\bar{r}_{1}^{0} t-\ln t+\bar{\theta}_{1}^{0}, \quad \bar{\theta}_{2}=\bar{r}_{2}^{0} t-2 \ln t+\bar{\theta}_{2}^{0} .
$$

All solutions of (4.6) are bounded with respect to $\bar{r}_{1}$ and $\bar{r}_{2}$. It is clear that $\bar{r}_{1} \rightarrow \bar{r}_{1}^{0}$ and $\bar{r}_{2} \rightarrow \bar{r}_{2}^{0}$ as $t \rightarrow \infty$. The orbital convergence of (4.8) to (4.7) means that $\bar{r}_{k}^{0}=r_{k}^{0}, k=1,2$ and there is an infinitely monotonically increasing function $s(t)$ such that

$$
\lim _{t \rightarrow \infty}\left(\bar{\theta}_{1}(t)-\theta_{1}(s(t))\right)=\lim _{t \rightarrow \infty}\left(\bar{\theta}_{2}(t)-\theta_{2}(s(t))\right)=0,
$$

or

$$
\begin{aligned}
& \lim _{t \rightarrow \infty}\left(r_{1}^{0} t-\ln t+\bar{\theta}_{1}^{0}-r_{1}^{0} s(t)-\theta_{1}^{0}\right)=0, \\
& \lim _{t \rightarrow \infty}\left(r_{2}^{0} t-2 \ln t+\bar{\theta}_{2}^{0}-r_{2}^{0} s(t)-\theta_{2}^{0}\right)=0 .
\end{aligned}
$$

From (4.9) we have $s(t)=t-\left(\ln t-\bar{\theta}_{1}^{0}+\theta_{1}^{0}\right) / r_{1}^{0}+o(1)$. Substituting this in (4.10) we obtain

$$
\lim _{t \rightarrow \infty}\left(\left(\frac{r_{2}^{0}}{r_{1}^{0}}-2\right) \ln t+\bar{\theta}_{2}^{0}-\theta_{2}^{0}+\left(\bar{\theta}_{1}^{0}-\theta_{1}^{0}\right) / r_{1}^{0}+o(1)\right)=0 .
$$

However, this is possible if and only if $r_{2}^{0}=2 r_{1}^{0}, \bar{\theta}_{1}^{0}=\theta_{1}^{0}, \bar{\theta}_{2}^{0}=\theta_{2}^{0}$. Consequently there are solutions of (4.6) which don't orbitally converge to any solution of (4.5).

Now we reformulate Theorem 4.3 for system (1.3).

Theorem 4.4. Let $x(t)$ be a bounded solution of (1.3) and

$$
t^{p}\|g(t, x(t))\| \rightarrow 0 \quad \text { as } \quad t \rightarrow+\infty
$$

with $p>1$. Then $x(t)$ approaches a certain invariant torus of unperturbed system (1.1).

Proof. It follows immediately from Theorem 4.3 and Section 3.

Note 4.7. For Theorem 4.4 Notes $4.4-4.6$ are also valid.

Example 4.4. The next example demonstrates that assumption $p>1$ cannot be generalized to include the case $p=1$. Let's consider again the two-dimensional linear centre (4.4) with the following perturbation

$$
\dot{x}_{1}=-x_{2}+\frac{\cos (\ln (\ln t))}{t \ln t} x_{1}, \quad \dot{x}_{2}=x_{1}+\frac{\sin (\ln (\ln t))}{t \ln t} x_{2}, \quad(t>1) .
$$

Obviously every bounded solution of (4.11) satisfies the conditions of Theorem 4.4 with $p=1$. But (4.11) has the bounded solutions

$$
x_{1}=r_{0} \cos t \exp (\sin (\ln (\ln t))), \quad x_{2}=r_{0} \sin t \exp (\sin (\ln (\ln t))),
$$

which oscillate between the circles with radii $r_{0} e$ and $r_{0} e^{-1}$. Hence no one of them can approach any circle $x_{1}=r \cos t, x_{2}=r \sin t$, which is just the solution of the unperturbed system. 


\section{The convergence of bounded solutions}

We begin with the main statement for system (1.5).

Theorem 5.1. Let (A3) or (A4) hold with $p>2$. Then each bounded solution of (1.5) converges to a certain solution of the unperturbed system (1.4).

Proof. Let $(r(t), \theta(t))$ denote any bounded solution of (1.5). Theorem 4.1 implies the convergence $r(t) \rightarrow r^{*}$ as $t \rightarrow+\infty$. Every solution of (1.4) lying on the invariant torus $r=r^{*}$ has the form $\left(r^{*}, A\left(r^{*}\right) t+\theta_{0}\right)$, where $\theta_{0}$ is the initial value. It is necessary and sufficient to prove the existence of a $\theta_{0}$ such that

$$
\lim _{t \rightarrow+\infty}\left(\theta(t)-A\left(r^{*}\right) t-\theta_{0}\right)=0 .
$$

Thus it is sufficient to prove that the limit value $\theta_{0}=\lim _{t \rightarrow+\infty}\left(\theta(t)-A\left(r^{*}\right) t\right)$ exists. Since

$$
\begin{aligned}
\theta(t) & -A\left(r^{*}\right) t=\theta(1)-A\left(r^{*}\right)+\int_{1}^{t}\left(\dot{\theta}(s)-A\left(r^{*}\right)\right) d s \\
& =\theta(1)-A\left(r^{*}\right)+\int_{1}^{t}\left(A(r(s))+Q(s, r(s), \theta(s))-A\left(r^{*}\right)\right) d s
\end{aligned}
$$

it is sufficient to prove that the obtained integral converges. By (A4) we have

$$
\left\|\left(\begin{array}{l}
P \\
Q
\end{array}\right)(s, r(s), \theta(s))\right\| \leq \alpha(r(s)) \beta(s) / s^{p}
$$

There is a constant $M$ such that $\alpha(r(s)) \beta(s) \leq M$ for $s>1$. Hence

$$
\left\|\int_{1}^{\infty} Q(s, r(s), \theta(s)) d s\right\| \leq M \int_{1}^{\infty} 1 / s^{p} d s=M /(p-1)
$$

Next we need to estimate $\left\|A(r(s))-A\left(r^{*}\right)\right\|$. First of all we notice that

$$
\begin{aligned}
& \left\|r(s)-r^{*}\right\|=\left\|\int_{+\infty}^{s} \dot{r}(u) d u\right\| \leq \int_{s}^{+\infty}\|P(u, r(u), \theta(u)) d u\| \\
& \quad \leq \int_{s}^{+\infty} \alpha(r(u)) \beta(u) / u^{p} d u \leq M \int_{s}^{+\infty} 1 / u^{p} d u=M s^{1-p} /(p-1) .
\end{aligned}
$$

Since $A \in \mathrm{C}^{1}$, it satisfies the Lipschitz inequality with some constant $L$ (on such a compact set $K$ from $\mathbf{R}_{+}^{m}$ that $r(s) \in K$ for $s \geq 1$ )

$$
\left\|A(r)-A\left(r^{*}\right)\right\| \leq L\left\|r-r^{*}\right\| .
$$

It follows from (5.2) and (5.3) that $\left\|A(r(s))-A\left(r^{*}\right)\right\|=O\left(s^{1-p}\right)$. Inequality $p>2$ implies convergence of $\int_{s}^{+\infty}\left\|A(r(s))-A\left(r^{*}\right)\right\| d s$. Hence limit

$$
\lim _{t \rightarrow+\infty}\left(\theta(t)-A\left(r^{*}\right) t\right)=\theta(1)-A\left(r^{*}\right)+\int_{1}^{\infty}\left(A(r(s))+Q(s, r(s), \theta(s))-A\left(r^{*}\right)\right) d s
$$

exists. This proves the theorem. 
Now we give the formulation of the analogous theorem for (1.3).

Theorem 5.2. Let (A1) or (A2) hold with $p>2$. Then each bounded solution of (1.3) converges to a certain solution of the unperturbed system (1.1).

Note 5.1. The condition $p>2$ cannot be generalized to include the case $p=2$ as the following example shows.

Example 5.1. Consider the system

$$
\dot{x}_{1}=-x_{2}^{3}, \quad \dot{x}_{2}=x_{1}^{3},
$$

and its perturbation

$$
\dot{x}_{1}=-x_{2}^{3}+\frac{1+\ln t}{(t \ln t)^{2}} x_{1}, \quad \dot{x}_{2}=x_{1}^{3}+\frac{1+\ln t}{(t \ln t)^{2}} x_{2} .
$$

The cycles of (5.4) are $x_{1}^{4}+x_{2}^{4}=C, C \in \mathbf{R}_{+}$. Let $(\mathrm{Cn} t$, Sn $t)$ denote the solution of (5.4) passing through point $(1,0)$ as $t=0$. Changing the variables by the formulas $x_{1}=$ $r \operatorname{Cn} \theta, x_{2}=r \operatorname{Sn} \theta$ (action-angle coordinates) we transform (5.4) into

$$
\dot{r}=0, \quad \dot{\theta}=r^{2},
$$

and (5.5) into

$$
\dot{r}=r \frac{1+\ln t}{(t \ln t)^{2}}, \quad \dot{\theta}=r^{2} .
$$

The solutions of (5.6) are $r(t)=r_{0}, \theta(t)=\theta_{0}+r_{0}^{2} t$ and those of (5.7) are $\bar{r}(t)=$ $r_{0} \exp [-1 /(t \ln t)], \bar{\theta}(t)=\theta_{0}+r_{0}^{2} \int_{2}^{t} \exp [-2 /(s \ln s)] d s$. Obviously, $\lim \bar{r}(t)=r_{0}$. But $\lim _{t \rightarrow+\infty}\left(\theta(t)-A\left(r_{0}\right) t\right)$ from proof of Theorem 5.1 does not exist. Indeed,

$$
\begin{gathered}
\lim _{t \rightarrow+\infty}\left(\theta(t)-A\left(r_{0}\right) t\right)=\lim _{t \rightarrow+\infty}\left(\theta(t)-r_{0}^{2} t\right)=\lim _{t \rightarrow+\infty} r_{0}^{2}\left(\int_{2}^{t} \exp [-2 /(s \ln s)] d s-t\right) \\
=\lim _{t \rightarrow+\infty} r_{0}^{2}\left(\int_{2}^{t}(\exp [-2 /(s \ln s)]-1) d s-2\right)=-\infty,
\end{gathered}
$$

because obtained integral diverges. This follows from the asymptotic equivalence

$$
\exp [-2 /(s \ln s)]-1 \sim-2 /(s \ln s)
$$

and divergence of $\int_{2}^{\infty} 2 /(s \ln s) d s$.

Note 5.2 (about convergency in Section 4). We wouls like to make an interesting remark about the possibility to decrease the lower bound in the inequality $p>2$ in Theorems 5.1 and 5.2. Suppose $A(r) \equiv A_{0}$ is a constant. For system (1.1) it is equivalent to $T_{k}\left(r_{k}\right) \equiv T_{k}$. It means that each subsystem $\dot{x}_{k}=f_{k}\left(x_{k}\right)$ in $(1.1), k=1, \ldots, n$, defines an isochronous centre. For (1.5) we can also talk about a property of isochronicity. Then the statements of Theorems 5.1-5.2 are valid for $p>1$. Indeed, we have in this case

$$
\lim _{t \rightarrow+\infty}\left(\theta(t)-A\left(r^{*}\right) t\right)=\theta(1)-A\left(r^{*}\right)+\int_{1}^{\infty} Q(s, r(s), \theta(s)) d s,
$$

where convergence of this integral follows from (5.1). 
The property of isochronicity in Hamiltonian systems (and not only for such systems)

is discussed in numerous papers. We refer only to [2], where further references can be found. Only for asymptotic perturbations of such systems, our Note 5.2 is valid.

We note that the main reason of appearance of the condition $p>2$ is just the necessity to estimate $A(r(t))-A\left(r^{*}\right)$. Hence we may suppose that $Q$ satisfies (A3) with $p>1$ (but $P$ with $p>2$ as before) and Theorem 5.1 will also be valid.

Finally we point out a possibility of the situation for system (1.1) when some subsystems of it are isochronous centres and all other not. It is not difficult to see that for "isochronous" angles $\theta_{k}$ the orbital convergence (Note: even usual convergence) is realized in Theorem 4.4 .

\section{Acknowledgements}

I am very grateful to the Royal Swedish Academy of Sciences for their financial support of this work. I would also like to thank G. Söderbacka for carefully reading this paper and his valuable remarks.

\section{References}

[1] Hartman P., Ordinary Differential Equations, John Wiley and Sons, New York, 1964.

[2] Cima A., Mañosas F. and Villadelprat J., Isochronicity for Several Classes of Hamiltonian Systems, J. Diff. Eqs, 1999, V.157, 373-413.

[3] Il'in Yu.A., Asymptotic Perturbation of a Nonlinear Center, (English. Russian original) Differ. Equations, 1997, V.33, N 5, 635-640; translation from Differents. Uravneniya, 1997, V.33, N 5, 1-6.

[4] Tabor M., Chaos and Integrability of Nonlinear Dynamics. An Introduction, A Wiley-Interscience Publication, John Wiley and Sons, N.Y., 1989.

[5] Wintner A., Asymptotic Equilibria, Amer. J. Math., 1946, V.68, 125-132.

[6] Wintner A., Asymptotic Integration Constants, Ibid., 1946, V.68, 553-559.

[7] Wintner A., Asymptotic Integration of the Adiabatic Oscillator, Ibid., 1947, V.69, 251-272.

[8] Yoshizawa T., Stability Theory and Existence of Periodic Solutions and Almost Periodic Solutions, Springer-Verlag, Applied Math. Sciences, V.14, 1975. 